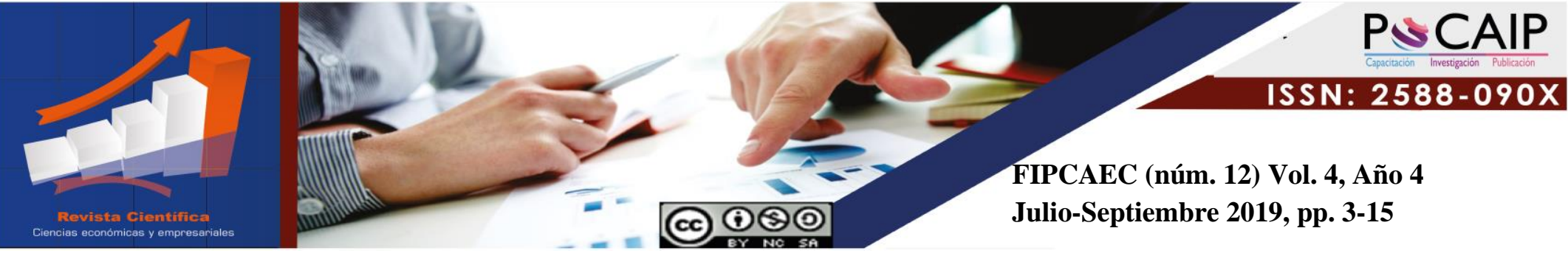

DOI: $10.23857 /$ fipcaec.v4i12.60

\title{
Análisis del modelo de diseño de puesto empresarial
}

\author{
Analysis of the business position design model
}

\section{Análise do modelo de design da posição de negócios}

\author{
Homero Manuel Ferrín-Schettini ${ }^{1}$ \\ homeromanuelferrin@hotmail.com \\ https://orcid.org/0000-0002-5876-4607
}

Correspondencia: homeromanuelferrin@hotmail.com

* Recepción: 12/ 04/ 2019 *Aceptación: 14/06/ 2019 *Publicación: 15 /07/ 2019

1 Doctor en Ciencias Administrativas, Magíster en Negocios Internacionales y Gestión de Comercio Exterior, Diploma Superior en Economía Internacional, Diploma Superior en Economía del Ecuador y del Mundo, Diploma Superior en Gestión Empresarial Internacional, Ingeniero Civil, Arquitecto, Docente de la Universidad Laica Eloy Alfaro de Manabí, Manta, Ecuador. 


\section{Resumen}

La investigación tuvo por objetivo analizar el modelo de diseño de puesto de la empresa Eurofish S.A., con la finalidad de conocer su funcionamiento en razón de generar conclusiones que permitan el abordaje en el mediano plazo de un plan estratégico para el fortalecimiento de la empresa. Teniendo una tipología descriptiva, la población estuvo conformada por 25 empleados de la empresa objeto de estudio, a quienes se les aplicó un instrumento de 21 ítems en escalamiento de Likert de cinco alternativas, con un coeficiente de Alfa de Cronbach de 0,871 catalogándose como muy alta. El trabajador utiliza distintas habilidades y conocimientos, diversos equipamientos y procedimientos para ejecutar tareas diferentes. La empresa posee un $45 \%$ de deficiencia en relación a la correspondencia entre el perfil del puesto de trabajo y lo ejecutado por el talento humano, siendo esto en detrimento de la organización.

Palabras clave: Toma de decisiones; cambio organizacional; administración pública; gestión de recursos.

\section{Abstract}

The objective of the research was to analyze the position design model of the company Eurofish S.A., with the purpose of knowing its operation by generating conclusions that allow the medium-term approach of a strategic plan for the strengthening of the company. Having a descriptive typology, the population was made up of 25 employees of the company under study, to whom an instrument of 21 items in Likert scaling of five alternatives was applied, with a Cronbach's alpha coefficient of 0.871 being classified as very high. The worker uses different skills and knowledge, various equipment and procedures to perform different tasks. The company has a $45 \%$ deficiency in relation to the correspondence between the profile of the job and the work carried out by human talent, this being detrimental to the organization.

Keywords: Decision-making; organizational change; public administration; resource management. 
Resumo

O objetivo da pesquisa foi analisar o modelo de design de posição da empresa Eurofish S.A., com o objetivo de conhecer sua operação, gerando conclusões que permitem a abordagem de médio prazo de um plano estratégico para o fortalecimento da empresa. De tipologia descritiva, a população era composta por 25 funcionários da empresa em estudo, aos quais foi aplicado um instrumento de 21 itens na escala Likert de cinco alternativas, sendo classificado como muito alto um coeficiente alfa de Cronbach de 0,871. O trabalhador utiliza diferentes habilidades e conhecimentos, vários equipamentos e procedimentos para executar tarefas diferentes. A empresa possui uma deficiência de $45 \%$ em relação à correspondência entre o perfil do trabalho e o trabalho realizado pelo talento humano, prejudicando a organização.

Palavras-chave: Tomada de decisão; mudança organizacional; administração pública; Gerenciamento de recursos

\section{Introducción}

Las organizaciones nacen con el fin de lograr diversos objetivos que de manera individual no podrían ser alcanzados por las personas, sean éstas de carácter público o privado. Al respecto, Chiavenato (2002, p. 7) menciona que una organización "es un sistema de actividades conscientemente coordinadas, formado por dos o más personas, cuya cooperación recíproca es esencial para la existencia de aquella". Por lo tanto, una organización sólo existe cuando hay personas capaces de comunicarse y están dispuestas a actuar conjuntamente, para obtener un objetivo en común. De esto se deduce que una organización es un medio por el cual dos o más personas de forma consciente y voluntaria realizan acciones recíprocas para lograr un fin preestablecido, las cuales llevan a cabo a través de la comunicación, trae como consecuencia la permanencia y desarrollo de la misma.

En la organización las personas en retribución a su labor deben recibir beneficios, así como herramientas que le faciliten su trabajo y que incrementen su eficiencia laboral, por lo que las organizaciones cuentan con un área de recursos humanos que debe contribuir al desarrollo del personal en todos sus niveles, con el objetivo de maximizar la eficiencia (Chiavenato 2002). 
Por lo antes dicho, las empresas deben contar con un área que se encargue exclusivamente de administrar el recurso humano dentro de ellas, la administración de recursos humanos (ARH) asume esta área en las organizaciones, la misma desarrolla cinco subsistemas que comprenden: Admisión, Aplicación, Compensación, Desarrollo, Mantenimiento y Monitoreo de Personas. De ellos se derivan una serie de procesos vitales para toda organización: el reclutamiento, la selección, capacitación, la evaluación de desempeño, el diseño de puesto, entre otros.

En ese mismo orden de ideas, es importante aclarar que los trabajos que realizan las personas en las organizaciones son los elementos esenciales de todas las estructuras organizacionales. De hecho, la razón de ser de las organizaciones es permitir que los individuos realicen el trabajo en los puestos que se les asigna. La expresión organicémonos significa, que necesitamos aclarar el trabajo que debe hacer cada individuo. Pero también nos interesa la ejecución eficaz de las labores, y necesitamos entender las causas del desempeño laboral eficaz e ineficaz. Una de las causas importantes del buen desempeño laboral es el diseño del puesto, lo que obtenemos al aclarar lo que debe hacer cada empleado (Ivancevich 2006, p. 174).

En relación al diseño del puesto Ivancevich (2006, p. 174) "se refiere al proceso por el cual los administradores deciden las labores y la autoridad del puesto en lo individual". Para ello, hay que tomar en cuenta las consideraciones muy prácticas que se asocian con el diseño del puesto, es decir, los aspectos vinculados con la efectividad en términos económicos, políticos y monetarios, por consiguiente es evidente su importancia en términos sociales y psicológicos.

En correspondencia con antes expresado, los puestos de trabajo pueden ser fuente de estrés psicológico e incluso de deterioro mental y físico. En un sentido más positivo, los puestos de trabajo proporcionan ingresos, experiencias de vida significativas, autoestima, estimación por los demás, regulación de la vida propia y asociación con los demás. Por tanto, el bienestar de las organizaciones y las personas guarda relación con la eficacia con que la dirección de una empresa diseñe sus puestos de trabajo (Ivancevich, 2006).

Ahora bien, para diseñar un puesto de trabajo, se necesita de la información sobre los puestos y los requisitos para llenarlos, los cuales se obtienen a través de un proceso denominado análisis de 
puestos, en el cual los analistas de puestos recaban la información sobre diferentes trabajos de manera sistemática, la evalúan y organizan. Un documento que ayuda al análisis de puestos es el organigrama con el cual opera la institución. Éste permite conocer de manera gráfica la ubicación de cada puesto, su interrelación y las líneas jerárquicas y de comunicación, muestra la estructura organizativa de las unidades administrativas de una organización, sus relaciones, la clasificación oficial de sus funciones y la jerarquía que les corresponde (Werther y Davis, 2008, p. 91).

Por su parte Bohlander (2001, p. 99), afirma, el diseño de puesto es "la estructuración de los puestos con miras a mejorar la eficiencia de la organización y la satisfacción laboral de los empleados". El diseño de puesto lo que busca es cambiar, modificar y enriquecer los puestos a efectos de captar los talentos de los empleados, al mismo tiempo que pretende mejorar el desempeño laboral de los empleados y de la organización.

Tomando como referencia lo planteado anteriormente, se hace indispensable desarrollar un análisis del modelo de diseño de puesto para optimizar el desempeño del talento humano de la empresa Eurofish S.A., ubicada en La Pradera de la ciudad de Manta - Ecuador, debido al alcance e importancia que tiene el cumplimiento de sus metas en mercado regional y nacional, resultado que les permitirá ubicarse en los niveles de excelencia en el mundo de los negocios, para lograr ese propósito los miembros de la organización, deben tener bien definido su diseño de puesto de trabajo y a su vez impulsados hacia un desempeño laboral optimo, de tal manera que esta influya en la productividad de la organización, es así que la investigación tiene por objetivo analizar el modelo de diseño de puesto de la empresa Eurofish S.A., con la finalidad de conocer su funcionamiento en razón de generar conclusiones que permitan el abordaje en el mediano plazo de un plan estratégico para el fortalecimiento de la empresa.

\section{Desarrollo}

\section{Modelado del trabajo}

La forma en que las personas trabajan en las organizaciones depende básicamente de la forma en que su trabajo se planeó, modeló y organizó, es decir de la manera en que se distribuyeron las tareas. En general, los puestos contienen las tareas y la estructura de los puestos la condiciona el 
diseño organizacional en el que está contenida. Los puestos forman parte integral del formato estructural de la organización (Chiavenato, 2009, p. 202).

No obstante, en muchas organizaciones todavía está intacto el concepto de puesto, numerosos órganos de recursos humanos, aún analizan y describen los puestos como en el pasado. Un puesto constituye una unidad de la organización el cual consiste en un conjunto de deberes y responsabilidades que lo separan y diferencian de los demás puestos (Chiavenato, 2009, p. 203).

\section{Descripción de los puestos}

Describir un puesto significa relacionar lo que hace el ocupante, cómo lo hace, en qué condiciones y por qué. Al respecto Chiavenato (2009, p. 222), afirma que "la descripción del puesto es un retrato simplificado del contenido y de las principales responsabilidades del puesto. El formato común de una descripción del puesto incluye el título del puesto, el resumen de las actividades que serán desempeñadas y las principales responsabilidades”.

Desde esta perspectiva la descripción del puesto relaciona, de forma breve, las tareas, las obligaciones y las responsabilidades del puesto. Es narrativa y expositiva y se ocupa de los aspectos intrínsecos del puesto, es decir, de su contenido. Una vez hecha la descripción de los puestos viene el análisis, el cual aborda los aspectos extrínsecos del puesto, es decir, cuáles son los requisitos que el ocupante debe cumplir para desempeñar el puesto.

\section{Concepto de análisis de los puestos}

Analizar un puesto significa detallar lo que exige de su ocupante en términos de conocimientos, habilidades y capacidades para que pueda desempeñar correctamente el puesto. El análisis se hace a partir de la descripción del puesto. Si bien están en estrecha relación, la diferencia radica en que la descripción se enfoca en el contenido del puesto (lo que el ocupante hace y cuándo, cómo y por qué lo hace) y el análisis de los puestos busca determinar cuáles son los requisitos físicos y mentales que el ocupante debe cumplir, las responsabilidades que el puesto le impone y las condiciones en que debe desempeñar el trabajo (Chiavenato, 2009, p. 222). 


\section{Diseño de puestos}

El diseño de puestos especifica el contenido de cada posición, los métodos de trabajo y las relaciones con los demás. Para Chiavenato (2009, p. 205), el diseño de puestos es la organización de las tareas y las actividades repetitivas de un puesto y las calificaciones que necesita el ocupante, así como su posición en la organización de trabajo como un todo. Por su parte Bohlander (2001, p. 99), afirma, el diseño de puesto es "la estructuración de los puestos con miras a mejorar la eficiencia de la organización y la satisfacción laboral de los empleados".

Desde esta perspectiva, el diseño de puesto busca cambiar, modificar y enriquecer los puestos a efectos de captar los talentos de los empleados, al mismo tiempo que pretende mejorar el desempeño de los empleados y de la organización, cada puesto exige ciertas competencias de su ocupante para que lo desempeñe bien. Esas competencias varían conforme al puesto, el nivel jerárquico y el área de actuación. Exigen que el ocupante sepa manejar los recursos, las relaciones interpersonales, la información, los sistemas y la tecnología con diferentes grados de intensidad.

El diseño de puestos es la manera en que cada uno se estructura y dimensión. Diseñar un puesto significa definir cuatro condiciones básicas:

- El conjunto de tareas o atribuciones que el ocupante debe desempeñar (cuál es el contenido del puesto).

- La manera en que las tareas o atribuciones se deben desempeñar (cuáles son los métodos y los procesos de trabajo).

- Con quién se debe reportar el ocupante del puesto (responsabilidad), es decir, quién es su superior inmediato.

- A quién debe supervisar o dirigir el ocupante del puesto (autoridad), es decir, quiénes son sus subordinados o las personas que dependen de él para trabajar (Chiavenato, 2009, p. 205).

Es importante que la empresa cuente con la debida definición de los puestos, esto permite generar un perfil organizacional, involucrándose la posibilidad de generar estrategias que permitan la 
optimización del talento humano en pertinencia de elevar la productividad, siendo considerable tener en consideración las características de quienes ocupan los distintos puestos con el fin de promover la concordancia entre talento humano y puesto de trabajo.

\section{Metodología de la investigación}

La investigación es de naturaleza descriptiva, porque se expone el evento estudiado enumerando detalladamente sus características, se registran, analizan e interpretan la totalidad de los datos suministrados por la muestra, Tamayo y Tamayo (2009), la describe como "un proceso sistemático de recolección de datos y análisis de información por medio de una descripción clara del proceso a estudiar" (p. 45), lo cual permitió describir la variable tal como se comporta en la realidad, sin manipulación alguna de la misma, lo cual le brinda a la investigación un diseño no experimental.

En cuanto a la población estuvo conformada por 25 empleados de la empresa objeto de estudio, a quienes se les aplicó un instrumento de 21 ítems en escalamiento de Likert de cinco alternativas, con un coeficiente de Alfa de Cronbach de 0,871 catalogándose como muy alta, teniéndose como técnica de análisis de información, la estadística descriptiva, la cual permitió establecer frecuencias y porcentajes para la descripción de la variable.

\section{Resultados}

Cuadro 1

Indicador Autonomía

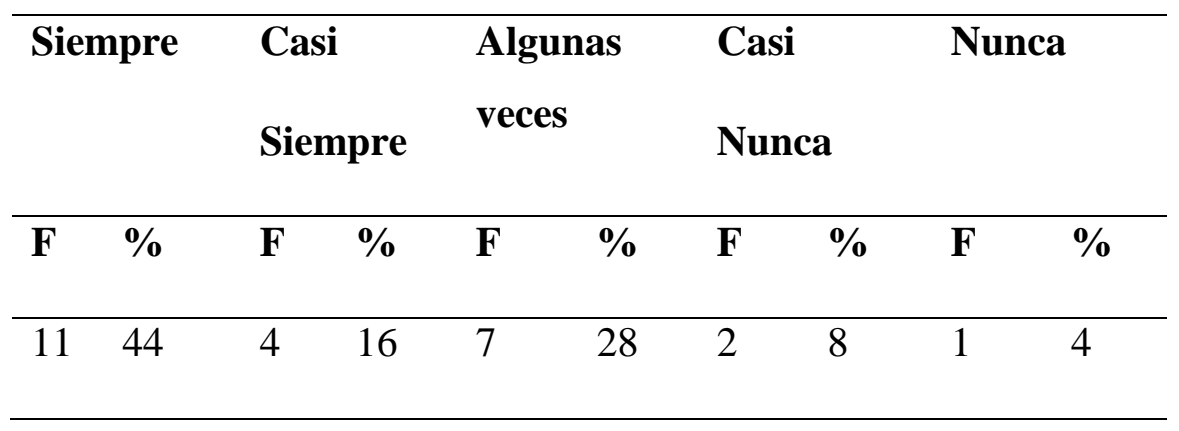

El $44 \%$ consideran que siempre el personal planifica y ejecuta sus actividades de trabajo con libertad, $16 \%$ casi siempre, $28 \%$ algunas veces, $8 \%$ casi nunca y $4 \%$ nunca respectivamente. 


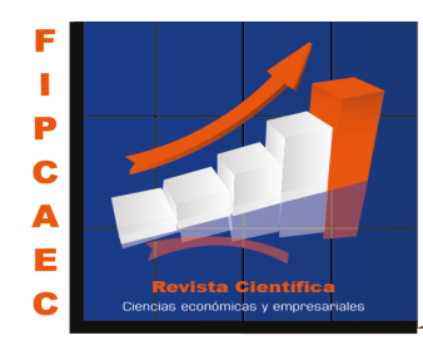

Ahora bien, al realizar un balance de ambos ítems, se determinó que el mismo tiene un comportamiento positivo. Al respecto, Bohlander (2001, p. 101) define la autonomía como el "grado en que puesto brinda libertad, independencia y discreción a la persona al calendarizar el trabajo y determinar los procedimientos que se usaran para realizarlo"

Cuadro 2

Indicador Significado

\begin{tabular}{llllllllll}
\hline Siempre & Casi & \multicolumn{2}{l}{$\begin{array}{l}\text { Algunas } \\
\text { veces }\end{array}$} & \multicolumn{2}{l}{ Casi } & & Nunca \\
& & Siempre & & & \\
& & & & & & & & \\
\hline F & $\%$ & F & $\%$ & F & $\%$ & F & $\%$ & F & $\%$ \\
\hline 11 & 44 & 7 & 28 & 6 & 24 & 1 & 4 & 0 & 0 \\
\hline
\end{tabular}

Los trabajadores opinaron que $44 \%$ que casi siempre Conoce el significado de las tareas que ejecuta, $28 \%$ casi siempre, $24 \%$ alguna veces y $4 \%$ casi nunca. Los aspectos antes descritos indican que los trabajadores Conocen el significado de las tareas que ejecuta. Lo expresado anteriormente coincide con el planteamiento de Chiavenato (2019), que dice "cuando más noción tiene el ocupante del significado de las tareas que ejecuta, mayor es la importancia que percibe en su trabajo, mayor su contribución y más elevada su responsabilidad".

Cuadro 3

Indicador Identidad

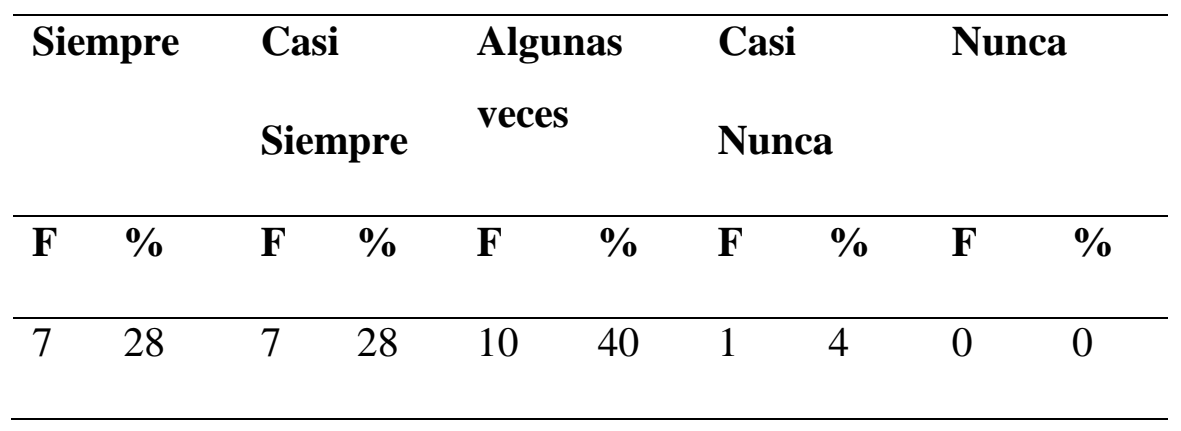


El 28\% de los trabajadores siempre realiza las actividades completa en su área de trabajo, 28\% casi siempre, $40 \%$ algunas veces y $4 \%$ casi nunca. De estos resultados se infiere que los trabajadores mantienen una identidad medianamente regular. Por ello, Chiavenato (2009), expresa "la identidad se relaciona con la posibilidad de la persona de efectuar un trabajo completo o global e identificarse con claridad los resultados de sus actividades" (p. 179).

Cuadro 4

Indicador Realimentación

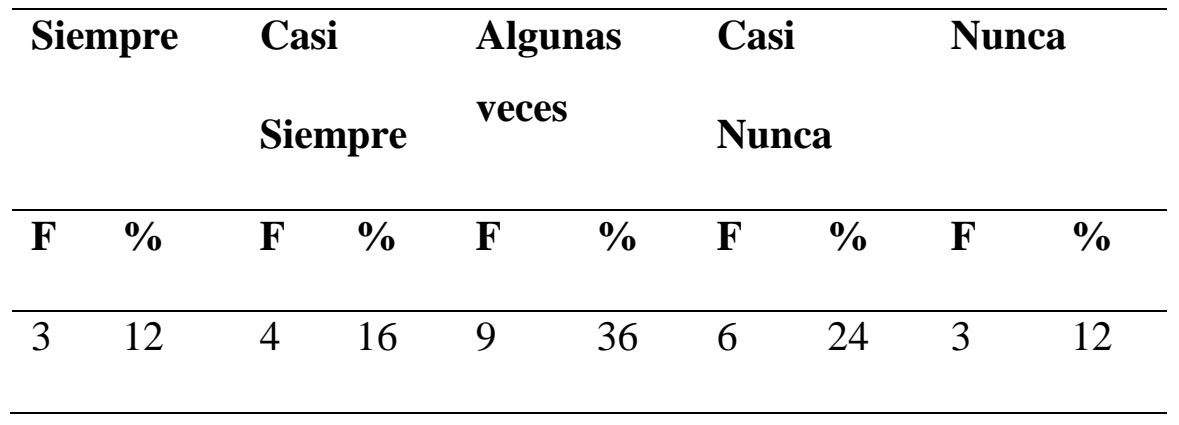

El 12\% de los trabajadores siempre recibe la información de los esfuerzos realizados en sus actividades, $16 \%$ casi siempre, $36 \%$ algunas veces, $24 \%$ casi nunca y $12 \%$ nunca. Los resultados anteriores demuestran que los trabajadores perciben como positivo la aplicación del indicador realimentación o retroalimentación. Con relación a esto último Bohlander (2001), considera que la aplicación de un adecuado proceso de retroalimentación conlleva a la ejecución de las actividades laborales requeridas por el puesto, que dan como resultado que el individuo reciba información clara y directa con respecto a la eficacia del desempeño.

Cuadro 5

Indicador Variedad

\begin{tabular}{llllllllll}
\hline Siempre & Casi & \multicolumn{2}{l}{$\begin{array}{l}\text { Algunas } \\
\text { veces }\end{array}$} & \multicolumn{2}{l}{ Casi } & \multicolumn{2}{l}{ Nunca } \\
& & \multicolumn{2}{l}{ Siempre } & & & & \\
& & & & & & & & \\
\hline F & $\%$ & F & $\%$ & F & $\%$ & F & $\%$ & F & $\%$ \\
\hline 11 & 44 & 8 & 32 & 4 & 16 & 2 & 8 & 0 & 0 \\
\hline
\end{tabular}




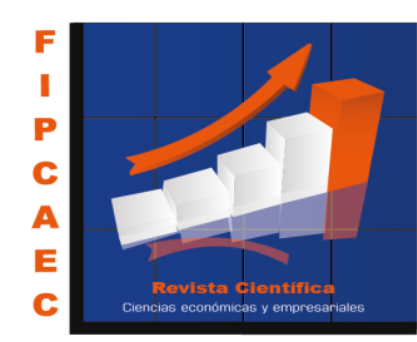

El $44 \%$ de los trabajadores, siempre realizan diversidad de habilidades en su puesto de trabajo, mientras que el $32 \%$ afirman que casi siempre, por su parte el $16 \%$ sostiene algunas veces y $8 \%$ casi nunca. Esta información permite determinar que el trabajador utiliza distintas habilidades y conocimientos, diversos equipamientos y procedimientos para ejecutar tareas diferentes. En forma general, estos resultados permiten afirmar que los trabajadores desarrollan diversidad de habilidades en su puesto de trabajo. Al respecto, Bohlander (2001) consideran que la variedad de habilidades es el "grado en que el puesto supone diversas actividades, que exigen el uso de varias habilidades y talentos diferentes por parte del ocupantes" (p. 101).

\section{Cuadro 6}

Indicador Autoestima

\begin{tabular}{|c|c|c|c|c|}
\hline Siempre & $\begin{array}{l}\text { Casi } \\
\text { Siempre }\end{array}$ & $\begin{array}{l}\text { Algunas } \\
\text { veces }\end{array}$ & $\begin{array}{l}\text { Casi } \\
\text { Nunca }\end{array}$ & Nunca \\
\hline$\%$ & $\%$ & $\%$ & $\%$ & $\mathbf{F}$ \\
\hline 8 & 2 & 20 & 20 & 11 \\
\hline
\end{tabular}

El 8\% respondió que siempre se siente motivado para desempeñar las tareas asignadas por su gerente inmediato, $8 \%$ casi siempre, $20 \%$ algunas veces, $20 \%$ casi nunca y $44 \%$ nunca. De los anteriores resultados se deduce, que el personal esta desmotivado para desempeñar las tareas asignadas por su gerente inmediato, de la misma forma trabaja en forma desmotivada en sus respectivos puestos de trabajos y no es reconocido dentro de todo el equipo de trabajo de la organización, por lo tanto los trabajadores poseen una autoestima baja, situación contraria lo planteado por Robbins (2004), quien considera “ que la autoestima se relaciona directamente con las esperanza de éxito, quienes tienen más autoestima creen que poseen la capacidad que requieren para triunfar en su empleo" (p. 100).

Por consiguiente de acuerdo a lo expresado por el autor, cuando la autoestima es más alta, los trabadores asumen más riesgos en la elección de los puestos de trabajos y eligen trabajos exigentes poco convencionales y de esta manera ayudan a alcanzar los objetivos 
organizacionales, los trabajadores con baja autoestima son más susceptibles a las influencias externas, buscan la aprobación de otros y sus actitudes está en correspondencia con los trabajadores que ellos respectan, es decir son complacientes con los demás.

\section{Conclusiones}

La empresa posee un $45 \%$ de deficiencia en relación a la correspondencia entre el perfil del puesto de trabajo y lo ejecutado por el talento humano, siendo esto en detrimento de la organización en consideración que baja el rendimiento del trabajador, así como se pueden estar realizando tareas que no se corresponden, generándose un desgaste físico, emocional, situación que conlleva a perder la calidad del servicio tal como plantean Aldana \& Piña (2017), conduciendo a pérdidas de recursos, con lo cual la efectividad de la empresa va en detrimento, siendo pertinente contrarrestar tal situación mediante la aplicación de estrategias operativas, así como afinar la filosofía de gestión en relación a los puestos de trabajo.

\section{Referencias}

1. Aldana, J., \& Piña, J. (2017). Calidad del servicio prestado al cliente por los instructores de gimnasios. Revista Arbitrada Interdisciplinaria Koinonía, 2(3), 172-197. Recuperado de http://fundacionkoinonia.com.ve/ojs/index.php/revistakoinonia/article/view/59/46

2. Bohlander G y Snell, S. (2001) Administración de Recursos Humanos. México. Duodécima edición. Internacional Thomson Editores

3. Chiavenato, I. (2002) Gestión del Talento Humano. Primera Edición. Portugal. MCGRAW- HILL INTERAMERICANA, S.A. Colombia.

4. Chiavenato, I. (2009) Gestión del Talento Humanos. Editorial. McGraw- HILL INTERAMERICANA., S.A. Colombia.

5. Ivancevich y otros. (2006). Comportamiento Organizacional. México. Sexta Edición. Editorial McGraw-Hill.

6. Robbins, S. (2004) Comportamiento Organizacional. Editorial Pearson. México. 


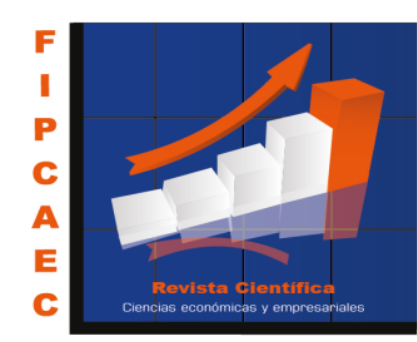

7. Tamayo y Tamayo (2009). El proceso de investigación Científica. Casos y práctica. México. Editorial Limusa.

8. Werther y Davis (2008). Administración de recursos humanos. El capital humano de las empresas. Sexta edición. México. Editorial McGraw-Hill.

\section{References}

1. Aldana, J., \& Piña, J. (2017). Quality of service provided to the client by gym instructors. Interdisciplinary Arbitrated Review Koinonía, 2 (3), 172-197. Recovered from http://fundacionkoinonia.com.ve/ojs/index.php/revistakoinonia/article/view/59/46

2. Bohlander G y Snell, S. (2001) Human Resources Administration. Mexico. Twelfth edition. Thomson International Publishers

3. Chiavenato, I. (2002) Human Talent Management. First edition. Portugal. MCGRAWHILL INTERAMERICANA, S.A. Colombia.

4. Chiavenato, I. (2009) Human Talent Management. Editorial. McGraw- HILL INTERAMERICANA., S.A. Colombia.

5. Ivancevich and others. (2006). Organizational behavior. Mexico. Sixth edition. McGrawHill editorial.

6. Robbins, S. (2004) Organizational Behavior. Pearson editorial. Mexico.

7. Tamayo and Tamayo (2009). The process of scientific research. Cases and practice. Mexico. Editorial Limusa.

8. Werther and Davis (2008). Human resources management. The human capital of companies. Sixth edition. Mexico. McGraw-Hill editorial.

\footnotetext{
(C2019 por el autor. Este artículo es de acceso abierto y distribuido según los términos y condiciones de la licencia Creative Commons Atribución-NoComercial-Compartirlgual 4.0 Internacional (CC BY-NC-SA 4.0)

(https://creativecommons.org/licenses/by-nc-sa/4.0/).
} 